\title{
Reducing Radiation Damage Using Pulsed Electron Beams in the TEM
}

\author{
Elisah J. VandenBussche ${ }^{1}$ and David J. Flannigan ${ }^{1^{*}}$ \\ 1. Department of Chemical Engineering and Materials Science, University of Minnesota, \\ Minneapolis, MN 55455, USA \\ * Corresponding author: flan0076@umn.edu
}

A rise in the development and the use of ultrashort-pulsed sources (i.e., femtosecond to picosecond) in transmission electron microscopes (TEMs) has led to numerous discoveries and paradigm tests of atomic to nanoscale structural dynamics well beyond the temporal limits of fast detectors [1,2]. Key to these advances are precise control over the electron emission process, typically via the photoelectric effect, or via temporal modulation of an initially continuous beam swept across an aperture [3]. In addition to enabling ultrafast pump-probe experiments, fine control over the emission process allows for exploration of the effects of such pulsed beams on damage sustained by the specimen during observation. Unlike proposed diffract-before-destroy methods with electrons [4,5], these approaches would ideally be applied in the single-electronper-packet regime, with sufficient time between the arrival of each electron at the specimen for all reversible energy-deposition events to fully recover. Indeed, some intriguing results using pulsed electron beams in TEMs have been reported, including effects on the structure of catalyst particles and apparent prolonged exposures for polymer crystals [6,7].

Here, we report the results of direct comparisons of the effects of pulsed and continuous electron beams on damage incurred by highly-sensitive specimens for identical dose rates and accumulated doses. The model system chosen for study was hexatriacontane $\left(\mathrm{C}_{36} \mathrm{H}_{74}\right)$, a longchain saturated hydrocarbon that forms well-ordered single crystals with low defect densities oriented along the [001] zone axis when drop-cast from solution onto a conductive amorphous carbon substrate. In addition to forming well-defined single crystals, the behaviors under continuous electron-beam illumination in conventional TEMs have been documented. Along with direct comparisons between pulsed and continuous beams, we also studied the effects of varying the time between each packet and the number of electrons in each packet. Overall, we measured a statistically-significant difference in the damage produced for pulsed, singleelectron beams compared to continuous beams delivered at the same dose rate for the same accumulated dose. The amount of damage incurred by the specimen as a function of accumulated dose, as quantified using Bragg-spot fading curves, was reduced by nearly a factor of two for the pulsed, single-electron beams as compared to continuous beams delivered at the same dose rate and with the same size illuminated area (within 1\%). This effect remained when the duration between the single-electron packets was reduced from $100 \mu \mathrm{s}$ to $5 \mu \mathrm{s}$, though the overall damage incurred was greater for both pulsed and continuous beams due to the higher dose rate $\left(9.0 \times 10^{-6} \mathrm{e} \cdot \AA^{-2} \cdot \mathrm{s}^{-1}\right.$ compared to $\left.7.8 \times 10^{-7} \mathrm{e} \cdot \AA^{-2} \cdot \mathrm{s}^{-1}\right)$. We also found that damage increased for the pulsed beams when more electrons per packet were used, and all benefits over continuous beams delivered at the same dose rates were lost when up to 20 electrons per packet were used ( $5 \mu$ s between packets). It is critical to note that all experiments were replicated at least once (up to five times for some), and all data was analyzed statistically with errors reported where possible. Our results indicate the highly-regular and high-precision delivery of electrons 
to the specimen via a pulsed beam enables statistically significant damage mitigation in the extreme-low-dose regime. Future work will focus on elucidating the mechanisms responsible for the observed effects and on exploring the prospects of increasing spatial resolutions [8].

[1] DA Plemmons et al., Chem. Mater. 27 (2015), p. 3178.

[2] A Feist et al., Ultramicroscopy 176 (2017), p. 63.

[3] W Verhoeven et al., Ultramicroscopy 188 (2018), p. 85.

[4] RF Egerton, Adv. Struct. Chem. Imaging 1 (2015), p. 5.

[5] JCH Spence, Struct. Dyn. 4 (2017), p. 044027.

[6] C Kisielowski et al., Adv. Funct. Mater. (2019), https://doi.org/10.1002/adfm.201807818

[7] OH Kwon et al., Proc. Natl. Acad. Sci. U.S.A. 108 (2011), p. 6026.

[8] This material is based on work supported by the U.S. Department of Energy, Office of

Science, Office of Basic Energy Sciences under Award No. DE-SC-0018204 and by the National

Science Foundation Graduate Research Fellowship Program under Grant No. DGE-00039202.

Partial support was provided by the Arnold and Mabel Beckman Foundation in the form of a Beckman Young Investigator Award.
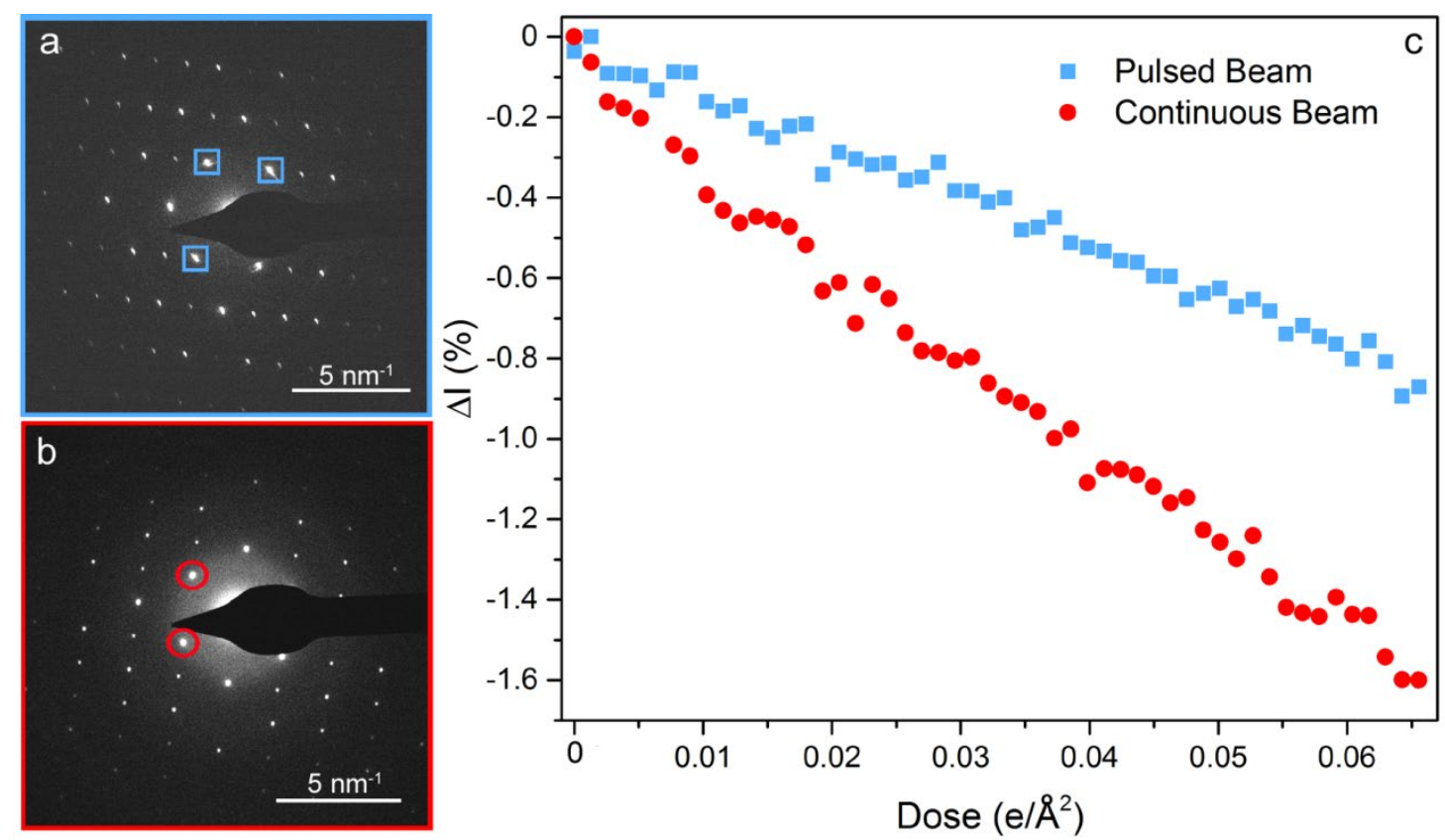

Figure 1. Pulsed compared to continuous electron-beam damage for a dose rate of $7.8 \times 10^{-7}$ $\mathrm{e} \cdot \AA^{-2} \cdot \mathrm{s}^{-1}$. Representative pulsed-beam (a) and continuous-beam (b) diffraction patterns of a $\mathrm{C}_{36} \mathrm{H}_{74}$ single crystal with the 110 Bragg spots used to generate the fading curves highlighted. (c) Fading curves for the pulsed and continuous beams. The slope of the continuous-beam data is steeper than that of the pulsed beam by approximately a factor of two. The pulsed-beam current and illuminated area were $1.6 \pm 0.24 \mathrm{fA}$ and $132.4 \mu \mathrm{m}^{2}$, respectively, while those of the continuous beam were $1.6 \pm 0.22 \mathrm{fA}$ and $131.5 \mu \mathrm{m}^{2}$, respectively. Error for the beam sizes is less than 1\%. The pulsed beam was generated with a 300-fs FWHM pulsed laser operated at 10 $\mathrm{kHz}$ (100 $\mu$ s between pulses), and the fluence impinging on the electron source in the TEM was adjusted so that the number of electrons in each packet was $1.03 \pm 0.15$ (error is one standard deviation). 